\title{
ESTUDIO EXPERIMENTAL DEL TIEMPO DE DISOLUCIÓN DEL RN-222 EN AGUA Y ALGUNAS IMPLICACIONES EN LA GEOLOGÍA
}

\author{
J. R. García-Vindas \\ Escuela de Física, Universidad de Costa Rica, \\ Sección de Física Nuclear Aplicada \\ Correo electrónico: jgarcia@ cariari.ucr.ac.cr
}

(Recibido 1/10/1999; Aceptado 8/2/2000)

\begin{abstract}
The study of radon like a tracer in Geology involves the study of radon's dissolution in water. The objective of this work is to study the time required to dissolve a volume of radon in water and to expel it from water. For this purpose a device has been built that allows dissolving or expelling a volume of radon in water. The results show that it is possible to dissolve or expel, in a short time, a volume of radon from water.

RESUMEN: El estudio de la disolución del radón en agua ha sido un tema de importancia en Geología, debido a las cualidades de este gas como trazador geoquímico. Para estudiar el tiempo de disolución y desgasificación de este gas, se utilizó un dispositivo que permite disolver y desgasificar una cantidad de radón en un volumen de agua. Los resultados muestran que es posible disolver y desgasificar rápidamente una cantidad de este gas en el agua.
\end{abstract}

\section{INTRODUCCIÓN}

La utilización de trazadores radiactivos en geología ha obtenido un amplio desarrollado en los últimos años. Cada vez se cuenta con mas técnicas aplicadas a la prospección de minerales y al estudio de la circulación de aguas subterráneas y superficiales. Uno de estos trazadores, utilizado recientemente, es el radón222, llamado comúnmente radón. Este gas radiactivo es producto de la desintegración del radio-226, es inerte y tiene una semivida de 3,8 días, lo cual permite que una cantidad de radón pueda recorrer largas distancias antes de desintegrarse por completo. El radón, que se encuentra naturalmente en el suelo, es utilizado en geología como precursor de eventos mayores, tales como sismos y erupciones volcánicas (King,1980; Wakita et al., 1989), indicador de fallas geológicas (Gates et al., 1990), en el campo de la geotermia, como indicador de zonas hidrotermales explotables (Whitehead et al., 1983) y en el campo de la hidrogeología, en el estudio de aguas subterráneas y superficiales (Rama \&Moore, 1984; Bonotto \& Andrews, 1997).

El radón que se produce por debajo del nivel freático, puede ser disuelto en el agua del manto freático, o ser desgasificado de ella con el paso de otros gases, tales como el $\mathrm{CO}_{2}, \mathrm{H}_{2} \mathrm{~S}$, etc. Este mecanismo de disolución y desgasificación juega un papel muy importante a la hora de utilizar el radón como trazador geoquímico. Es por ello que el estudio de la solubilidad del radón en el agua y su desgasificación en esta se vuelve importante.

Este trabajo muestra los resultados más relevantes de un conjunto de experiencias realizadas en el laboratorio de Geoquímica de la Universidad de Montpellier II, Francia, con el objetivo de estudiar el tiempo necesario para disolver una cantidad de radón, que atraviesa un volumen de agua, en dicha cantidad; así como también, el tiempo necesario para desgasificar o liberar el radón contenido en ella. 


\section{METODOLOGÍA}

Con el fin de comprender mejor la dinámica de disolución y desgasificación del radón en el agua, se realizó un conjunto de experiencias, a temperatura ambiente, con un dispositivo construido para disolver una cierta cantidad de radón en un volumen de agua conocido y de esta forma efectuar la desgasificación del mismo (Fig. 1). Este dispositivo esta compuesto de una cámara de detección 'A' de 3 litros, en la cual se instaló un detector de radón del tipo mencionado en Monnin \& Seidel, (1998), que mide la concentración del radón en la fase gaseosa. Un recipiente 'B' de 6 litros de volumen, se utilizó para contener una cantidad de agua conocida y dentro de la cual se instaló un tubo burbujeador que baja hasta el fondo del recipiente. Con este tubo se efectúa la disolución del radón en el agua del recipiente e igualmente la desgasificación del mismo. El recipiente 'B' se llena de agua casi por completo, de manera que el volumen de aire restante sea despreciable. El dispositivo también está compuesto de una bomba ' $C$ ' que efectuará la circulación del gas en todo el sistema, una cámara ' $\mathrm{D}$ ' que contiene pechblenda (fuente natural de radón) con una concentración de Rn-222 cercana a $1 \mathrm{MBq} / \mathrm{m}^{3}$, la cual suministra el radón al sistema y una bomba de vacío 'E', conectada a la fase gaseosa para evacuar el aire o el radón del sistema durante la experiencia.
En la figura 1 se muestra un conjunto de válvulas numeradas de 1 a 6 , las cuales tienen la función de aislar, cuando se necesite, las diferentes partes del dispositivo.

Con el fin de identificar mejor cada parte del procedimiento utilizado en el experimento se ha dividido éste en cuatro etapas principales llamadas: Admisión, Disolución, Evacuación y Desgasificación.

Etapa I. Admisión: Al principio del experimento se hace vacío en toda la fase gaseosa del sistema. Las válvulas 1 y 4 permanecen abiertas y todas las otras cerradas. Una vez que el vacío es obtenido, se cierra la válvula 1 y se abren las válvulas 2 y 6 , dejando siempre abierta la válvula 4. Con esto el radón es introducido al interior del recipiente por diferencia de presiones, formando así lo que llamaremos de aquí en adelante la fase gaseosa.

Etapa II. Disolución: Una vez que el equilibrio es alcanzado en la cámara de detección ' $\mathrm{A}$ ' (esto se verifica cuando la concentración de radón medida por el detector permanece casi constante), se cierran las válvulas 2 y 6 . Luego de esto se abre la válvula 3 y se pone en marcha la bomba ' $\mathrm{C}$ ' con un caudal de $0,5 \mathrm{l} / \mathrm{min}$ (válvulas $1,2,5$ y 6 cerradas). De esta manera, el radón contenido en la fase gaseosa burbujea en el agua del recipiente ' $\mathrm{B}$ ' y la cantidad de éste no disuelta

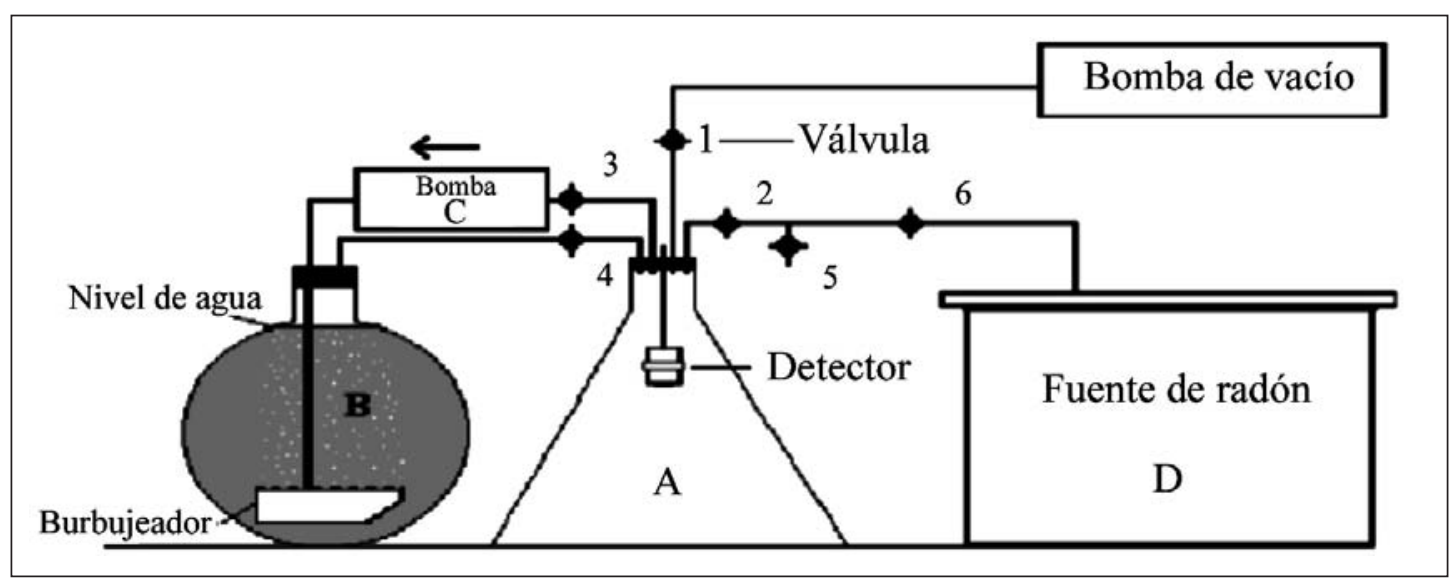

Fig. 1: Esquema del dispositivo utilizado en el experimento de disolución de radón en agua. 
en el agua pasa en circuito cerrado al recipiente 'A' para ser reinyectada de nuevo en el recipiente 'B'.

Etapa III. Evacuación: La duración de la etapa anterior es suficientemente larga, de tal manera que la concentración en la cámara de detección llegue a un valor casi constante. En este momento el equilibrio es alcanzado entre el radón de la fase gaseosa y el disuelto en el agua. Dicho de otro modo, no se puede disolver más radón en la cantidad de agua contenida en el recipiente 'B'. Las válvulas 3 y 4 se cierran ahora para luego abrir la 1, 2 y 5. La bomba de vacío se pone en marcha para extraer el radón contenido en la cámara de detección y reemplazarlo por aire, el cual es introducido dentro del recipiente 'A' por las válvulas 2 y 5. Esta última válvula comunica el sistema con la atmósfera por medio de un dispositivo de seguridad (no mostrado en la figura) con el fin de evitar la contaminación del laboratorio.

Etapa IV. Desgasificación: Para realizar la desgasificación se cierran las válvulas 1,2 y 5 , luego se abren las válvulas 3 y 4 (1, 2, 5 y 6 permanecen cerradas). Se pone en marcha la bomba 'C' con un caudal de $0,5 \mathrm{l} / \mathrm{min}$ para hacer burbujear el aire contenido en la cámara de detección
'A' y así provocar la desgasificación del radón en el agua del recipiente 'B'. Esta etapa persiste hasta que la concentración medida por el detector en la cámara 'A' permanezca casi constante.

\section{RESULTADOS}

Las figuras 2 y 3 muestran respectivamente dos de las experiencias más representativas entre todas las realizadas. Las dos últimas etapas, mencionadas en la sección anterior, se repitieron varias veces con el fin de efectuar desgasificaciones sucesivas.

Las gráficas de las figuras 2 y 3 están dividas en regiones para identificar cada proceso. Las regiones I, II, III y IV corresponden respectivamente con los procesos de Admisión, Disolución, Evacuación y Desgasificación. Nótese que III y IV se repiten, lo que indica que se realizaron desgasificaciones sucesivas. Cuando se realiza la etapa de evacuación, la lectura en el detector no alcanza el valor de cero debido a que posiblemente algunos átomos de Po-218, descendiente directo del radón, se fijan en el detector. Este elemento posee un semi-período de 3,05 min y se desintegra produciendo una partícula alfa cuya

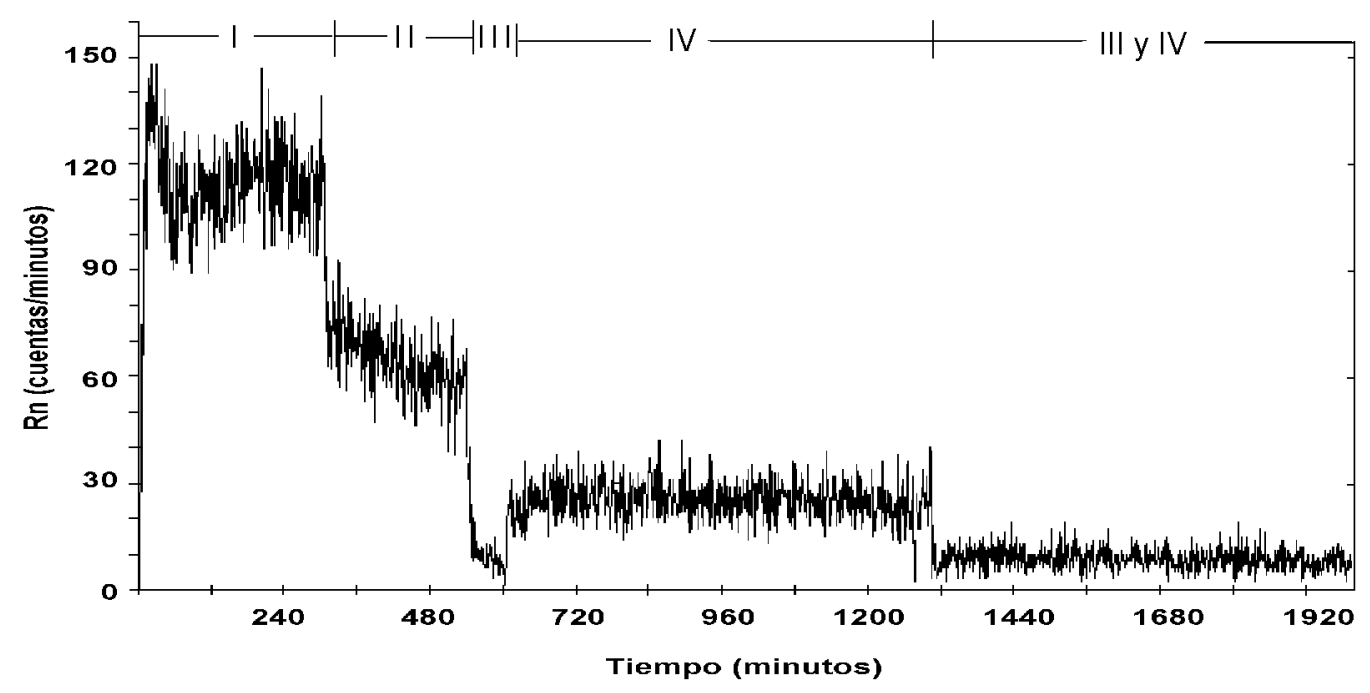

Fig. 2: Evolución de la concentración de radón en la cámara de detección durante el experimento. 


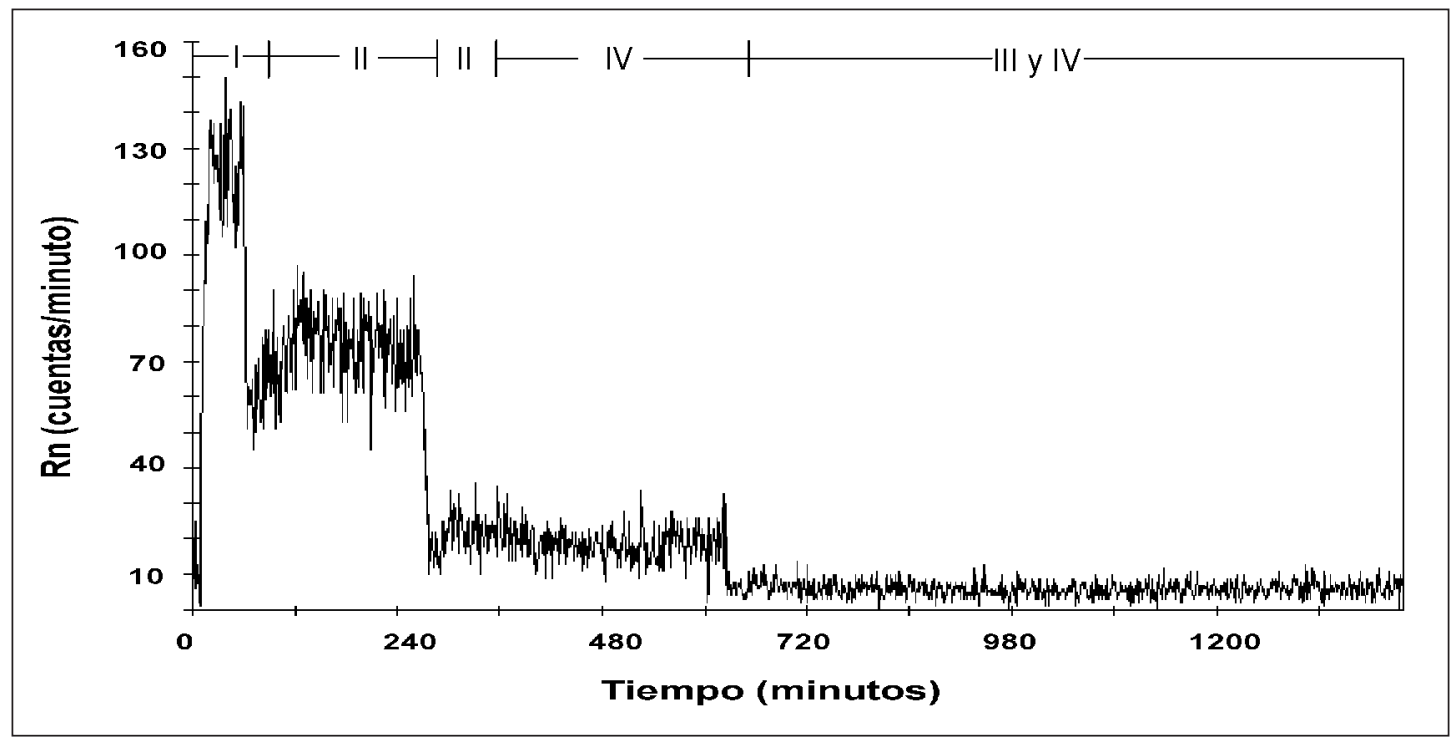

Fig. 3: Evolución de la concentración de radón en la cámara de detección durante el experimento.

energía es de $6 \mathrm{MeV}$. Esta energía es muy similar a la que posee la partícula alfa del radón $(5,48 \mathrm{Mev})$, por lo que el detector no puede discriminar entre ambas partículas, produciendo así un conteo diferente de cero. Este efecto se muestra en la etapa de evacuación de la figura 2 , comprendida después del minuto 480 y antes del minuto 600, donde el tiempo necesario para que el conteo en el detector alcance el valor de cero concuerda aproximadamente con el decaimiento del Po-218. Este efecto es constante en todas las etapas de evacuación y su contribución es despreciable, por lo que se decidió no tomarlo en cuenta para las experiencias posteriores (Figs. 2 y 3 ).

Durante la etapa de disolución, una cantidad de radón, representando aproximadamente el 30\% de la concentración inicial de la cámara de detección, es rápidamente disuelta en el agua, pero enseguida la disolución se vuelve más lenta. Este fenómeno se puede observar en las figuras 2 y 3, en la parte marcada II en ambas figuras. En las dos experiencias se observa que durante todo el proceso de disolución, la concentración de radón en la cámara de detección continua disminuyendo lentamente. Esto significa que él sigue disolviéndose durante todo este tiempo. La desgasificación (parte IV en las figuras) presenta una evolución similar a aquella encontrada en la disolución; una cantidad de radón es desgasificada rápidamente, aumentando la concentración en la cámara de detección. A diferencia de la disolución, la concentración alcanzada en la cámara de detección, durante los primeros minutos de la desgasificación, permanece constante durante todo el proceso. Esto se debe a que inicialmente se hace circular el aire de la cámara de detección a través del agua, pero debido a que el circuito de circulación es un circuito cerrado, el radón desgasificado se recupera en la cámara de detección y luego se envía nuevamente hacia el agua, por la bomba. A partir de este momento se cae en la etapa de disolución. La concentración en la cámara de detección alcanza el equilibrio cuando la relación de concentraciones entre la cámara de detección y el agua llega a ser igual a aquella obtenida en la etapa de disolución. Esta relación de concentraciones define lo que se conoce como coeficiente de disolución, el cual se expresa como:

$$
\mathrm{L}=\mathrm{C}_{\mathrm{w}} / \mathrm{C}_{\mathrm{g}}
$$


Donde $\mathrm{Cw}$ representa la concentración de radón en el agua y $\mathrm{Cg}$ la concentración de radón en la fase gaseosa. L varía con la temperatura de manera inversa. Si no se presentan cambios importantes de temperatura, $\mathrm{L}$ debe permanecer casi constante.

De los resultados obtenidos se desprende que, tanto el tiempo de disolución como el de desgasificación es del orden de algunos minutos. La figura 4 muestra una ampliación de la figura 2 en el intervalo de tiempo comprendido entre los 270 y 340 minutos. De esta figura se observa claramente que en 10 minutos, aproximadamente, la concentración en la fase gaseosa disminuye en un $30 \%$ de su valor inicial.

\section{CONCLUSIÓN E IMPLICACIONES DE LOS RESULTADOS OBTENIDOS}

Las implicaciones de los resultados obtenidos, sobre la interpretación de las medidas de la concentración de radón en el campo, son considerables.

En el caso de la disolución, los resultados obtenidos hacen concluir que una cantidad de agua exenta, o casi, de radón y circulando en el suelo puede disolver rápidamente una gran cantidad de este, disminuyendo así la concentración local del gas. Este fenómeno se encuentra por ejemplo cuando la lluvia cae sobre un suelo seco y permeable; el agua de lluvia, pobre en radón, se infiltra hacia abajo disuelve a su paso, rápida y eficazmente, una cantidad importante de radón y la lleva a niveles inferiores del suelo. Este fenómeno fue verificado experimentalmente por el autor (García-Vindas, 1999).

Otro ejemplo de esto se presenta con las aguas de los acuíferos. En muchos casos el agua del acuífero recorre distancias del orden de metros antes de llegar a la naciente. Si la velocidad de circulación de esta permite que el radón transportado por ella, en disolución, no se desintegre por completo antes de llegar a la naciente, entonces se podrá encontrar una cantidad importante de radón en este lugar (Andrews \& Lee, 1979; Lawrence et al., 1991). De aquí que los acuíferos deban ser considerados sitios de interés a la hora de efectuar mediciones de la concentración de radón, pues estos pueden llevar más rápidamente la señal geoquímica a la superficie, donde generalmente se instalan los equipos de medición.

En el caso de la desgasificación, su corta duración trae consecuencias importantes. La zona que se encuentra por debajo del nivel freático presenta generalmente grandes concentraciones de radón debido a que este se encuentra disuelto en el agua. El paso de gases, tales como el $\mathrm{CO}_{2} \mathrm{O}$ el $\mathrm{H}_{2} \mathrm{~S}$, a través del nivel freático en una zona hidrotermal o volcánica, por ejemplo, causará la rápida desgasificación de una gran cantidad de radón (Gasparini \& Mantovani, 1978; Ribeiro \&

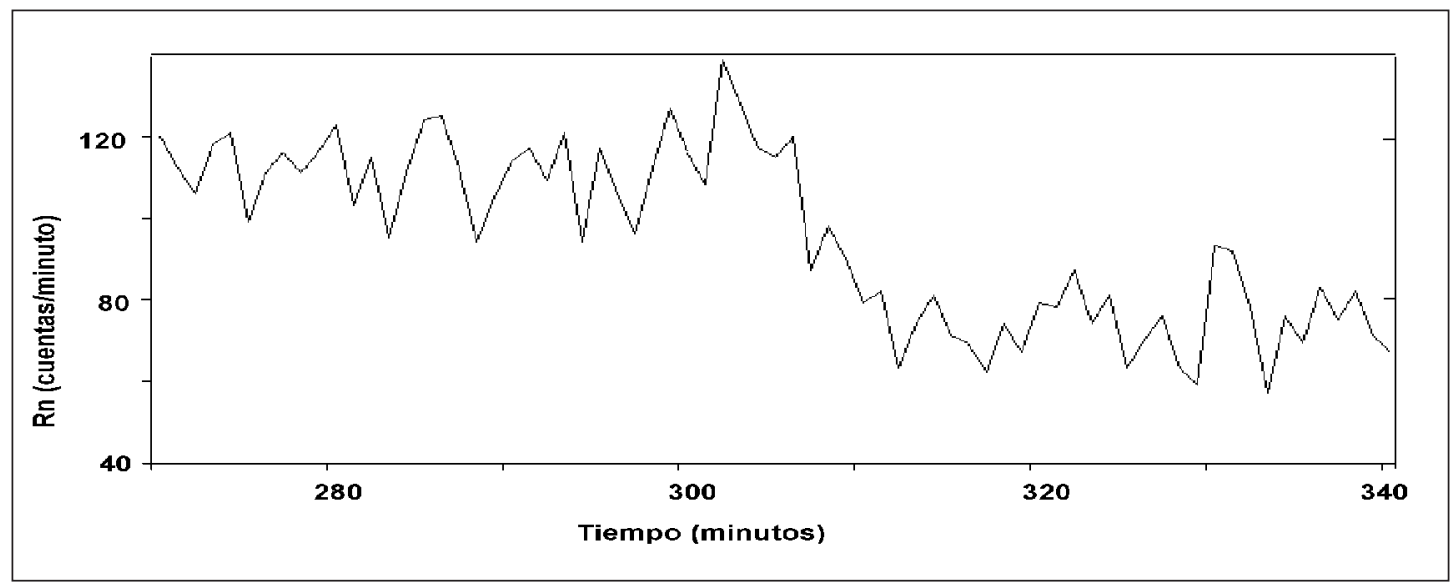

Fig. 4: Ampliación de la figura 2 en el intervalo comprendido entre 270 y 340 minutos. 
Mantovani, 1984). Si la cantidad de $\mathrm{CO}_{2}$ o de $\mathrm{H}_{2} \mathrm{~S}$ es importante y su desgacificación es de una duración suficiente, este podrá evacuar todo el radón y llevarlo hacia las capas superiores del suelo, aumentando la concentración local en este nivel.

De los resultados de este trabajo se infiere también, que los acuíferos son zonas de gran interés para la medición del gas radón, en el marco de la predicción de eventos sísmicos y volcáni$\cos$, ya que éste es transportado rápidamente hacia la superficie, en el caso de la desgasificación, reflejando de este modo la evolución geoquímica del lugar.

\section{REFERENCIAS}

ANDREWS, J.N. \& LEE, D.J., 1979: Inert gases in groundwater from the bunter sandstone of England as indicators of age and palaeoclimatic trends. - J. Hydrol. 41: 233-252.

BONOTTO, D.M. \& ANDREWS, J.N., 1997: The implications of laboratory Rn-222 flux measurements to the radioactivity in groundwaters: the case of karstic limestone aquifer. - Appl. Geochem. 12: 715-726.

GARCÍA-VINDAS, J.R., 1999: Transport du radon en milieu poreux (experimentation et modelisation) : implication pour la realisation et l'interpretation de mesures in situ. -277 págs. Université de Montpellier II. [Tesis Ph.D].

GASPARINI, P. \& MANTOVANI, M.S.M., 1978 : Radon anomalies and volcanic eruptions. - J. Volcanol. Geotherm. Res. 3: 325-341.

GATES, A.E., GURDENSEN, L.C.S. \& Malizzi, L.D., 1990: Comparison of radon in soil over faulted crystalline terranes: glaciated versus unglaciated. - Geophys. Res. Lett. 17: 813-816.

KING, C.Y., 1980: Episodic radon changes in subsurfaces soil gas active faults and possible relation to earthquakes. - J. Geophys. Res. 85: 3065-3078.

LAWRENCE, E., POETER, E. \& Wanty, R., 1991: Geohydrologic, geochemical, and geologic controls on the occurrence of radon in ground water near Conifer, Colorado, USA. - J. Hydrol. 127: 367-386.

MONNIN, M. \& SEIDEL, J., 1998: An Automatic Radon Probe for Earth Science Studies. J. Appl. Geophys. 39: 209-220.

RAMA \& MOORE, W.S., 1984: Mechanism of transport of U-Th series radioisotopes from solids into ground water. - Geochimica Cosmochimica Acta 48: 395-399.

RIBEIRO, F.B. \& MANTOVANI, M.S.M., 1984: Determinaçao dos Parâmetros de Difusao do Radônio em Funçao da Temperatura para Alguns Minerais e Rochas, e suas Implicaçoes en Ambientes Volcanicos. - An. Acad. Brasil. Ciênc. 56: 184-196.

WAKITA, H., IGARASHI, G., NAKAMURA, Y., SANO, Y. \& NOTSU, K., 1989: Coseismic radon changes in groundwater. Geophys. Res. Lett. 16: 417-420.

WHITHEAD, N.E., GINGRLCH, J.E. \& FISCHER, J.C., 1983: A New Zealand test of the track-etch method of prospecting for geothermal steam. - J. Volcanol. Geotherm. Res. 15: 339-354. 